\title{
An Optimized Fingerprinting-Based Indoor Positioning with Kalman Filter and Universal Kriging for 5G Internet of Things
}

\author{
Shuai Huang $\mathbb{D}^{1,2}$ Kun Zhao $\mathbb{D}^{1,2}$ Zhengqi Zheng, ${ }^{1,2}$ Wenqing Ji, ${ }^{1,2}$ Tianyi Li, $^{3}$ \\ and Xiaofei Liao ${ }^{4}$ \\ ${ }^{1}$ Engineering Center of SHMEC for Space Information and GNSS, East China Normal University, Shanghai 200241, China \\ ${ }^{2}$ Shanghai Key Laboratory of Multidimensional Information Processing, East China Normal University, Shanghai 200241, China \\ ${ }^{3}$ Shanghai Red Center, Ericsson, Shanghai 310000, China \\ ${ }^{4}$ School of Information Science and Technology, Donghua University, Shanghai 201620, China
}

Correspondence should be addressed to Kun Zhao; kzhao@ce.ecnu.edu.cn

Received 4 April 2021; Revised 25 May 2021; Accepted 4 June 2021; Published 19 June 2021

Academic Editor: Fa Zhu

Copyright (C) 2021 Shuai Huang et al. This is an open access article distributed under the Creative Commons Attribution License, which permits unrestricted use, distribution, and reproduction in any medium, provided the original work is properly cited.

Fingerprinting technique for indoor positioning based on $5 \mathrm{G}$ system has attracted attention. Kalman filter (KF) is used as preprocessing of raw data to reduce the disturbance of Received Signal Strength (RSS) values. After preprocessing, Universal Kriging (UK) algorithm is adopted to reduce the efforts of establishing a fingerprinting database by Spatial Interpolation. A machine learning algorithm named $K$-Nearest Neighbour (KNN) is used to calculate user equipment's position. Real experiments are setup with 5G signals over the air. Two indoor scenarios are considered depending whether the base station is located in the same room with user equipment or not. In test room A, the proposed KF and UK algorithms achieve 53\% positioning accuracy improvement. In test room B, $43 \%$ performance improvement is obtained by the proposed algorithm. $1.44-$ meter positioning error is observed as the best case for $80 \%$ test samples.

\section{Introduction}

Global Navigation Satellite System (GNSS) has provided enough accuracy for outdoor positioning but not good indoor. 5G Internet of Things (IoT) is a popular research topic including various application scenarios such as indoor positioning, smart transportation, smart manufacturing, and smart security [1-4]. A variety of indoor positioning systems have emerged, including Ultra-Wide Band (UWB), WiFi, Bluetooth, and Long-Term Evolution (LTE) [5-7]. The Base Stations (BSs) of LTE are widely distributed, which has shown advantages for IoT, Machine Learning (ML), and edge intelligence. 5G New Radio (NR) continues to evolve to further enhance LTE performance [8-12]. The number of connected devices in $5 \mathrm{G}$ is increasing rapidly and continues to grow exponentially.

Reference Signal Receiving Power (RSRP), Received Signal Strength (RSS), Sounding Reference Signal (SRS) and other signals are used for positioning [13-15]. RSS-based positioning system includes a radio propagation distance loss model and fingerprinting method $[16,17]$. The radio propagation distance loss model requires multiple BSs to perform trilateral positioning and applies in simple environments, while it is not easy to observe multiple NR BSs in a room in the early deployment phase. Hence, we choose the fingerprinting technique in this paper. Fingerprinting technique includes offline and online stages. In the offline stage, NR, RSS, and coordinates of each reference point are extracted to form fingerprints and input into a fingerprinting database. In the online stage, the RSS of the test point is measured in real time and compared with the offline fingerprints to calculate positions. It is important to build a reliable fingerprinting database

Varying multipath, Non-Line-of-Sight (NLOS) always makes RSS biased and reduces the reliability of fingerprints. To solve the problem, preprocessing methods are introduced 
to mitigate multipath effects. Reference [18] proposes a method that reduces the effect of signal multipath fading in RSS-distance estimation using Kalman filter. Zhang et al. proposed an indoor positioning method combining MEMS sensors and wireless fingerprints. They used Kalman filter to constrain WIFI fingerprints, which can improve positioning accuracy and computational efficiency [19]. Besides, constructing offline fingerprints requires a lot of manpower and resources. Spatial interpolation methods are considered to improve the spatial resolution of fingerprints with less manual efforts. In [20], Zuo et al. proposed a time-variant multiphase fingerprint map indoor localization method based on Kriging interpolation. Reference [21] introduces a variant of inverse distance weight (IDW) interpolation which is a Modified Shepard method. Son et al. proposed Universal Kriging interpolation based on drift function [22]. This method showed a better performance than linear interpolation, inverse distance weighing, and Ordinary Kriging. Intelligent fingerprinting techniques widely use machine learning as the algorithm to calculate the positions of things [23]. A novel multimodal complete tracking system based on statistic and DL techniques is presented by reference [24]. The authors used a multiphase statistical fingerprint and deep learning to estimate target indoor position. In [25], KNN method was used to achieve the position based on RSS data received by the module to be located. And the authors used $\mathrm{KF}$ to optimize the positioning information.

In this paper, Kalman filter $(\mathrm{KF})$ is used as preprocessing optimization method. Specifically, it consists of two stages. In the offline stage, the raw RSS is filtered to obtain reliable data. In the online stage, the RSS collected in real time can be filtered to eliminate the influence of varying multipath. We use spatial interpolation to interpolate the fingerprinting database and compare a variety of interpolation methods including Universal Kriging (UK) to improve the resolution of fingerprints. K-Nearest Neighbour (KNN) algorithm is taken as the positioning algorithm.

\section{System Model}

In the positioning system, we collect RSS signal and use the signal to calculate location of the mobile phone. As shown in Figure 1, our positioning system consists of two stages, offline and online. In the offline stage, we collect the RSS signal of the reference point and build the raw fingerprint database. After RSS preprocessing, we can build a RSS preprocessed fingerprinting database. By performing spatial interpolation on the database, we can build a database that is reliable and accurate. In the online stage, we capture the RSS signal of the test point and preprocess the signal. And we use the positioning algorithm to determine the location of the test point.

\section{RSS Preprocessing}

Kalman filter is a linear minimum variance estimation algorithm. As shown in Figure 2, KF algorithm consists of a gain calculation loop and a filter calculation loop. The gain calculation loop includes filter gain, estimation error, and predic- tion error. The filter calculation loop includes state prediction and state estimation.

The covariance of the observation noise $R$ is represented by averaging the variance of the RSS at each reference point. The phone remains stationary during the observation at a point. Set the system process noise $Q$ equals to 0.001 , the state transition vector $\Phi$ equals to 1 , and observation vector $H$ equals to 1 . During RSS filtering of the reference points, the first estimated error covariance of the point is obtained as

$$
P_{1}=\frac{1}{N} \sum_{l=1}^{N}\left(Z_{1}^{l}-E\left[Z^{l}\right]\right)^{2} \text {, }
$$

where $Z_{1}^{l}$ is the first sample RSS of point $l$. $E\left[Z^{l}\right]$ denotes mathematical expectation of RSS data of the point $l . N$ is denoted by the number of reference points. The following is calculated for point $l$. The prediction error covariance of the $t$ th sample RSS is expressed as

$$
P_{t, t-1}^{l}=P_{t-1}^{l}+Q
$$

where $P_{t-1}^{l}$ represents the $(t-1)$ th sample estimated error covariance. Filter gain of the $t$ th sample RSS, denoted by $J_{t}^{l}$, is

$$
J_{t}^{l}=P_{t, t-1}^{l}\left[P_{t, t-1}^{l}+R\right]^{-1}
$$

The estimated error covariance of the $t$ th sample RSS is expressed as

$$
P_{t-1}^{l}=\left[I-J_{t}^{l}\right] P_{t, t-1}^{l}\left[I-J_{t}^{l}\right]^{T}+J R J_{t}^{l^{T}}
$$

where $I$ denote unit vector. The predicted value of the $t$ th RSS is expressed as

$$
X_{t, t-1}^{l}=\Phi X_{t-1}^{l}
$$

We put the filter gain into the filter calculation loop to get the estimated value of the $t$ th sample of RSS

$$
X_{t}^{l}=X_{t, t-1}^{l}+J_{t}^{l}\left[Z_{t}^{l}-X_{t, t-1}^{l}\right]
$$

where $Z_{t}^{l}$ is the $t$ th sample of RSS.

Through the KF, the error generated by the RSS can be reduced in the measurement process so that we can obtain more accurate RSS. For the fingerprinting database, a more accurate offline fingerprinting database is established. At the same time, we ensure RSS real-time accuracy for the points to be located.

\section{Spatial Interpolation}

When we build the offline fingerprinting database in the fingerprinting positioning system, within a certain resolution range, the positioning accuracy is proportional to the resolution of the offline fingerprinting database. The increase of 




FIGURE 1: Flow chart of fingerprint indoor positioning method.

resolution will lead to a substantial increase in workload. To reduce the time cost while ensuring the positioning accuracy, the spatial interpolation method is used to effectively and correctly improve the resolution of offline fingerprints and reduce the workload. The spatial interpolation method obtains RSS values of interpolation points from those of reference points in the area. As shown in Figure 3, the solid one is an interpolation point, and the hollow ones are the reference points.

In the actual scene, the neighbours of linear interpolation and IDW have a great influence on the result. To solve this problem, we consider Kriging interpolation algorithm. The Ordinary Kriging requires RSS value of point $l$ to meet the second-order stability which is $E\left[X^{l}\right]=C$, where $C$ is constant. However, NR RSS signal cannot satisfy this assumption in indoor room, which means $E\left[X^{l}\right]=m\left(x_{l}, y_{l}\right)$ is a nonstationary function of the spatial position. Universal Kriging uses a deterministic drift function and residual function to express the RSS value, and the RSS value at any point $t$, denoted by, $X^{l}$, is:

$$
X^{l}=m\left(x_{l}, y_{l}\right)+r\left(x_{l}, y_{l}\right)
$$

where $m\left(x_{l}, y_{l}\right)$ represents the drift function of NR RSS related to the position coordinate $\left(x_{l}, y_{l}\right), r\left(x_{l}, y_{l}\right)$ is the residual function of NR RSS expected to be zero. $m\left(x_{1}, y_{l}\right)$ is used to describe the trend of RSS. And we use a deterministic function to simulate it. According to the distribution characteristics of RSS in two-dimensional space, $m\left(x_{l}, y_{l}\right)$ is expressed by a quadratic function [26]:

$$
m\left(x_{l}, y_{l}\right)=\sum_{i=0}^{L} \alpha_{i} f_{i}\left(x_{l}, y_{l}\right)=\alpha_{0}+\alpha_{1} x_{l}+\alpha_{2} y_{l}+\alpha_{3} x_{l}^{2}+\alpha_{4} x_{l} y_{l}+\alpha_{5} y_{l}^{2},
$$

where $\alpha_{i}$ is the coefficient of the deterministic function $f_{i}\left(x_{l}, y_{l}\right)$ and $L$ equals 5 . The weight coefficient of UK not only depends on the distance between the interpolation point and reference points but also is related to NR RSS of distribution characteristics in the space. The semivariogram $\gamma(d)$ is related to the distance $d$ between each

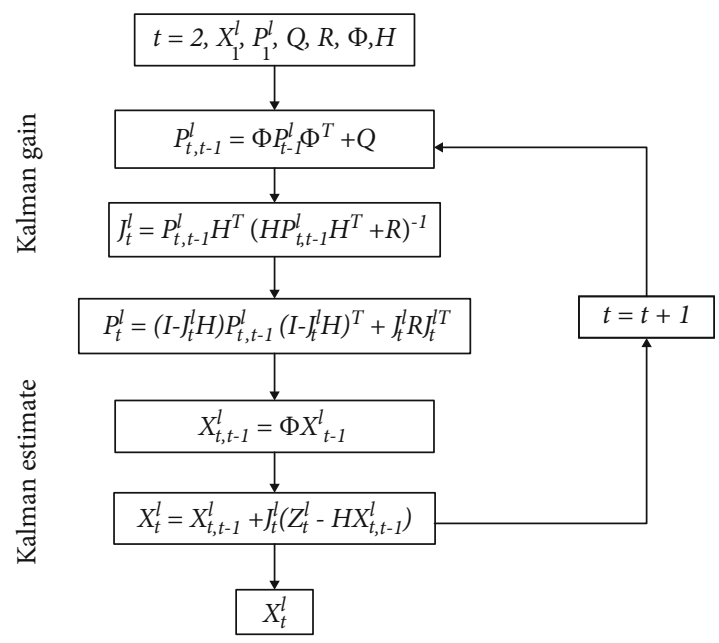

FIgURE 2: Kalman filter structure diagram.

point in space. The semivariogram is equal to half of the mathematical expectation of the square of the difference between NR and RSS of all points separated by a given interval $d$.

We fit the semivariogram by the RSS value of the known points. Commonly used variation function models include exponential model, spherical model, Gaussian model, and multifunction model. Spherical model is used to fit the function model which has good stability and robustness. The spherical model is defined as

$$
\gamma(d)= \begin{cases}0 & |d|=0, \\ c_{0}+c\left(\frac{3 d}{2 a}-\frac{d^{3}}{2 a^{3}}\right) & 0<|d|<a, \\ c_{0}+c & |d| \geq a,\end{cases}
$$

where $c_{0}, c$, and $a$ are the coefficient of the semivariogram $\gamma(d)$.

UK algorithm is unbiased and optimal estimation. Unbiasedness means that the expected value of the estimator is equal to the true value. The optimal estimator means that the estimator has the smallest variance among all such linear 
unbiased estimators. We need to obtain the weight coefficient $\lambda$ of each reference point, which is defined as

$$
\sum_{u=1}^{g} \lambda_{u}=1
$$

$\lambda_{u}$ is the weight coefficient of the point $u$. Using Lagrange multiplier method to solve the weight coefficient matrix U:

$$
\left[\begin{array}{c}
\mathbf{U} \\
\mathbf{F}
\end{array}\right]=\left[\begin{array}{ll}
\mathbf{W} & \mathbf{S} \\
\mathbf{S}^{T} & \mathbf{O}
\end{array}\right]^{-1}\left[\begin{array}{l}
\mathbf{V} \\
\mathbf{G}
\end{array}\right],
$$

where $\mathbf{S}$ represents the coordinate function matrix of the reference point as

$$
\left[\begin{array}{ccc}
f_{0}\left(x_{1}, y_{1}\right) & \cdots & f_{L}\left(x_{1}, y_{1}\right) \\
\vdots & \ddots & \vdots \\
f_{0}\left(x_{g}, y_{g}\right) & \cdots & f_{L}\left(x_{g}, y_{g}\right)
\end{array}\right]
$$

$\mathbf{U}$ is the weight coefficient matrix of reference points as $\left[\begin{array}{lll}\lambda_{1} & \cdots & \lambda_{g}\end{array}\right]^{T}$. G denotes the coordinate function matrix of the interpolation point $h$ which is $\mathbf{G}=$ $\left[f_{1}\left(x_{h}, y_{h}\right) \quad \cdots \quad f_{L}\left(x_{h}, y_{h}\right)\right]^{T}$. W represents the variation function matrix between reference points as

$$
\left[\begin{array}{ccc}
\gamma_{1,1} & \cdots & \gamma_{1, n} \\
\vdots & \ddots & \vdots \\
\gamma_{n, 1} & \cdots & \gamma_{n, n}
\end{array}\right]
$$

$\mathbf{V}$ denotes the variation function matrix between the reference point and interpolation point which is $\mathbf{V}=$ $\left[\begin{array}{lll}\gamma_{1, h} & \cdots & \gamma_{n, h}\end{array}\right]^{T}$. $\mathbf{F}$ is denoted by the Lagrange coefficient matrix $\left[\begin{array}{lll}\eta_{0} & \cdots & \eta_{L}\end{array}\right]^{T}$, where $\eta$ is Lagrange coefficient. $\mathbf{O}$ is the $(L+1) *(L+1)$ matrix of zeros.

Then, we get

$$
X^{*}(h)=\sum_{u=1}^{g} \lambda_{u} X(u),
$$

where $X^{*}(h)$ is the RSS estimated value at the $h$ th interpolation point, $X(u)$ is NR RSS value of the reference point $u, g$ is the number of reference points of the interpolation point $h$.

\section{Experimental Results and Discussion}

Indoor positioning is an indispensable part of human life in the future. Due to the different locations of base stations and the diversity of indoor rooms, indoor positioning in different rooms is considered.

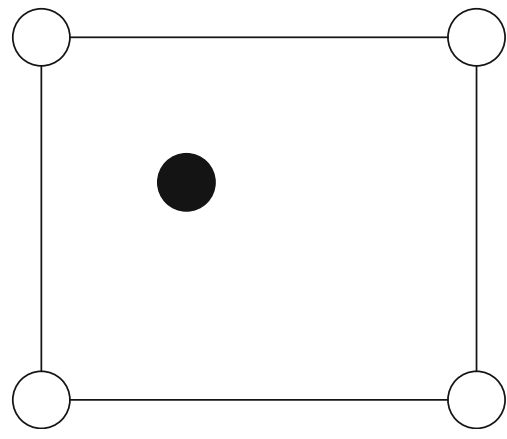

FIGURE 3: Spatial interpolation graph.

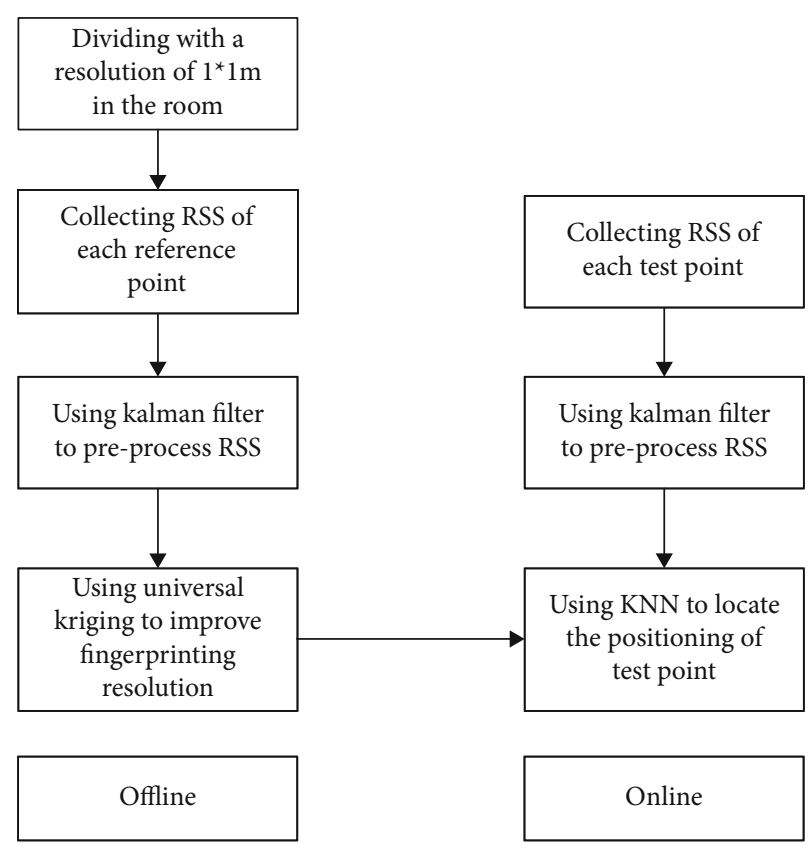

FIGURE 4: Fingerprinting positioning structure.

5.1. Experimental Setup. The experimental system adopts a single 5G base station with fingerprinting offline and online stages as shown in Figure 4.

Positioning accuracy is affected by the placement of BS. This experiment is performed in two different indoor rooms as shown in Figure 5. There were people walking around during measurements. Solid dots are fingerprinting reference points, the hollow ones are spatial interpolation points, and those stars are test points. 5G test phone model is Samsung S20 G9810, and 5G BS is Nokia Airscale 5G Small Cell.

The BS and room A are located in the same room (as shown in Figure 5(a)). The BS is set up in the corner and 3.5 meters high above the floor. The mobile phone is placed on a one-meter tall tripod. The L-shaped room is divided into 21 squares with a resolution of $1 \mathrm{~m} * 1 \mathrm{~m}$. In indoor fingerprinting positioning, please note that higher resolution will greatly increase the workload of establishing offline fingerprints. And due to the complexity of the environment, the resolution is chosen to fit for the distribution characteristics of RSS in the room. During the experiments, we analyze the RSS data, which remains stable during two minutes. Hence, 




(a)

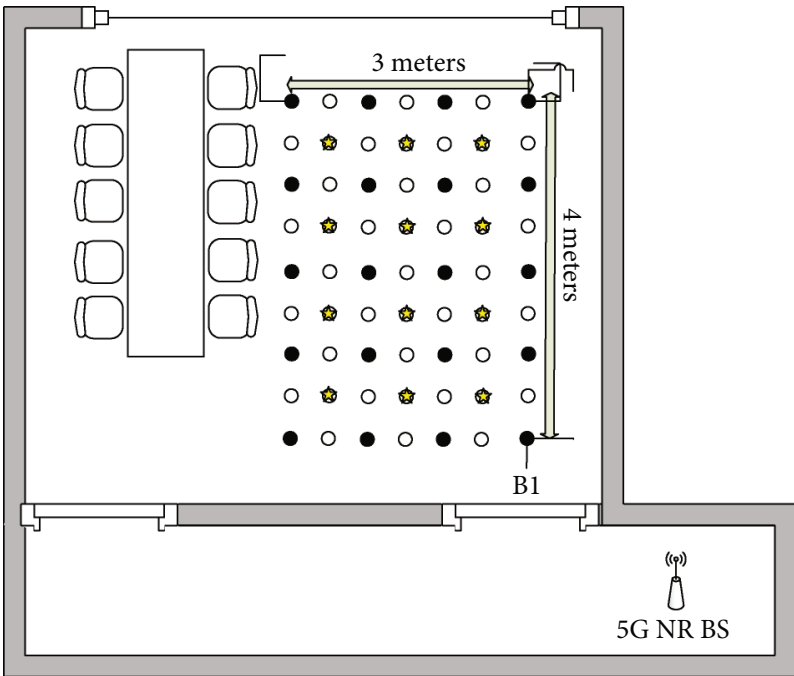

(b)

Figure 5: (a) Positioning room A. (b) Positioning room B.

we do not measure more time to keep our time. We randomly select 8 test points in the positioning area and statically collect RSS data at 34 reference points and 8 test points for 2 minutes, and the fetch rate of RSS is $100 \mathrm{~ms} / \mathrm{sam}$ ple. The inherited value from the last moment is used when raw data is lost. We perform spatial interpolation in room A with a resolution of $0.5 \mathrm{~m} * 0.5 \mathrm{~m}$.

The BS is set up 3.62 meters high above the floor in the corridor adjacent to the room as in room B as shown in Figure 5(b). The doors between them are closed. Mobile phone is placed on a one-meter tall tripod. The distribution of reference points and interpolation points is the same as that of room A. The test points are put in the centre of each grid.
KNN regression algorithm is used as the positioning algorithm in the experiments. In KNN, samples with higher similarity are mapped to close distances. The estimated position is the average of the coordinates of the nearest neighbours.

Positioning error is defined as

$$
e=\frac{1}{M_{k}} \sum_{k=1}^{M_{k}} \sqrt{\frac{1}{M_{b}} \sum_{b=1}^{M_{b}}\left\|q_{k}-q \wedge_{k}(b)\right\|^{2}},
$$

where $q_{k}$ denotes ground-truth of test point $k . \hat{q}_{k}(b)$ is the estimated position based on the $b$ th sample of test point $k$. 


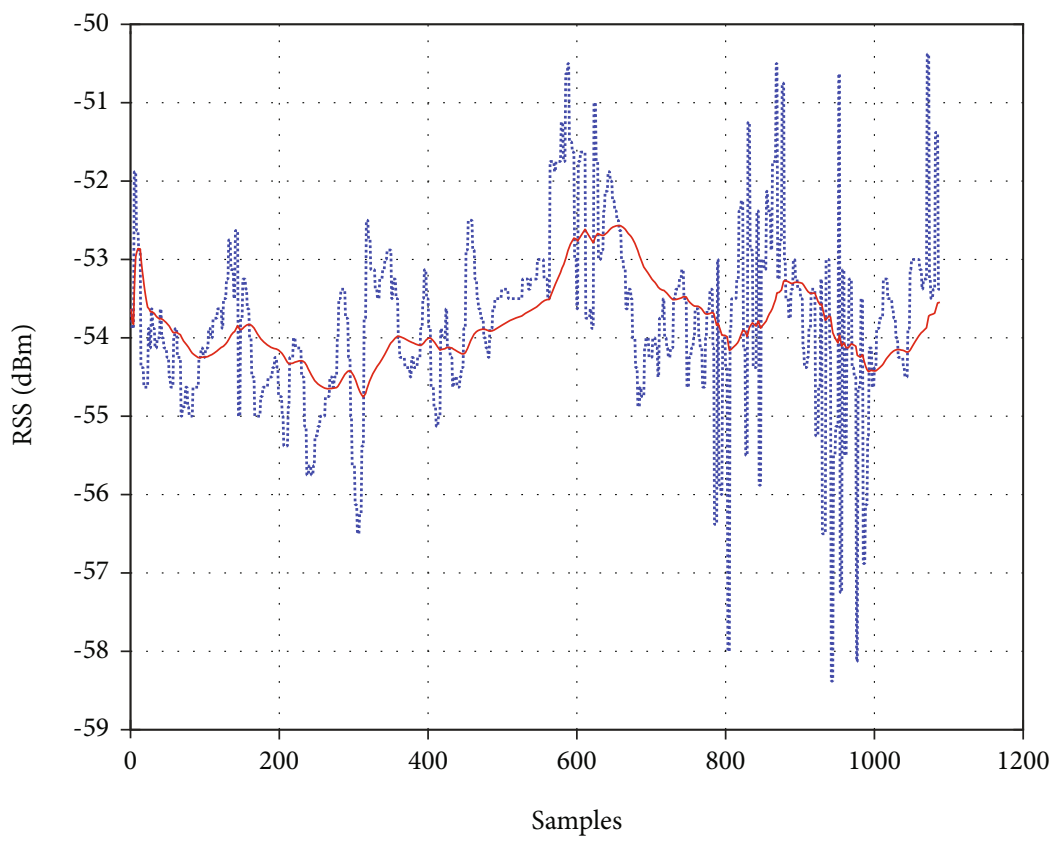

...... Raw data

— KF data

(a)

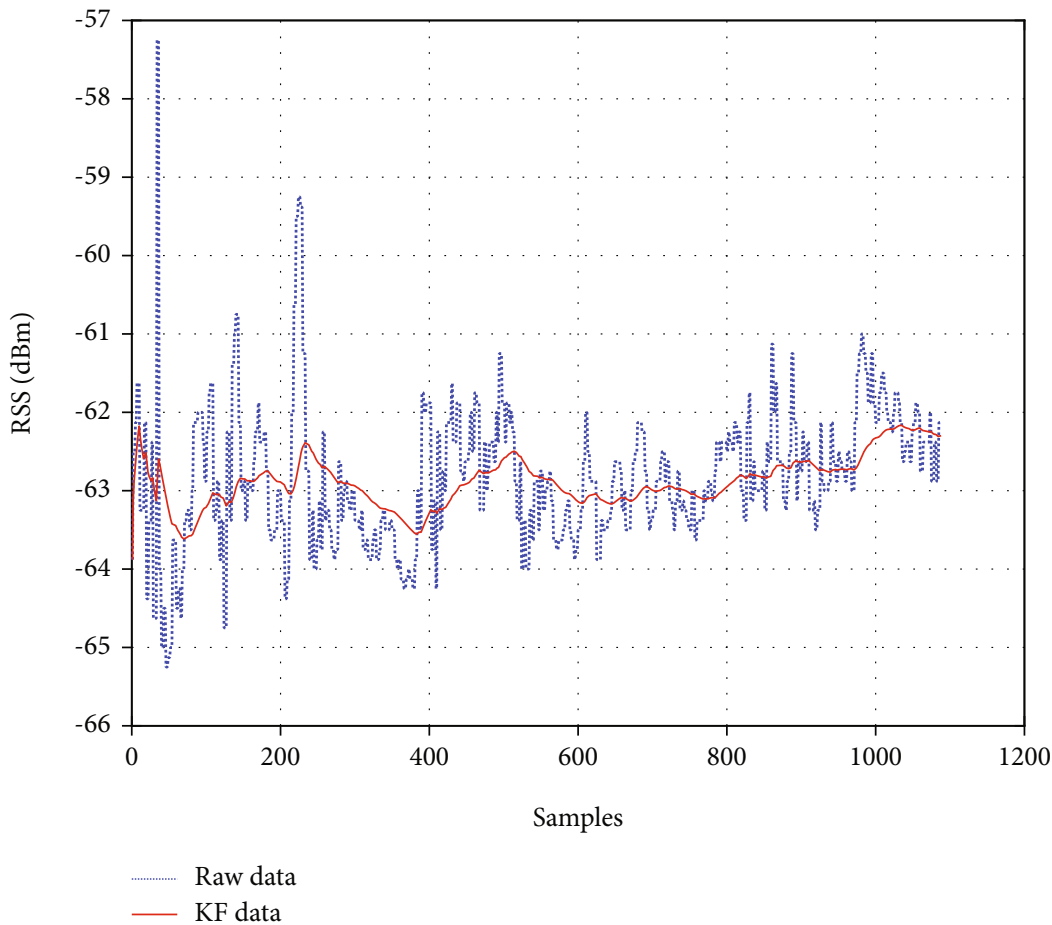

(b)

FIGURE 6: (a) RSS comparison chart before and after Kalman filter of reference point A1 in room A. (b) RSS comparison chart before and after Kalman filter of reference point B1 in room B. 


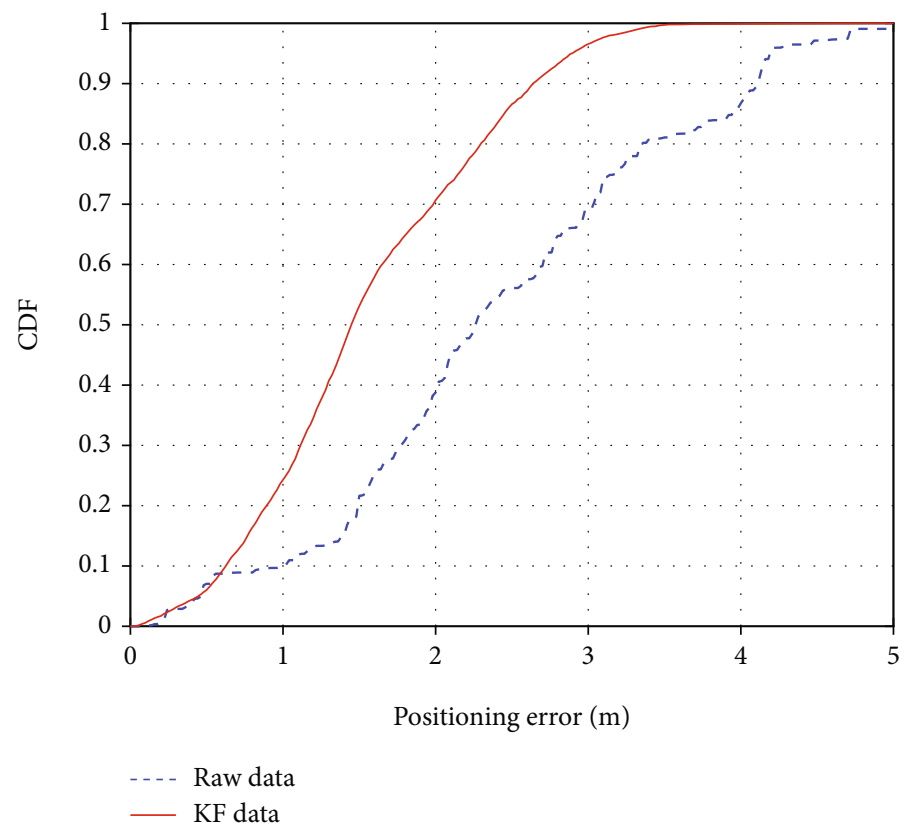

(a)

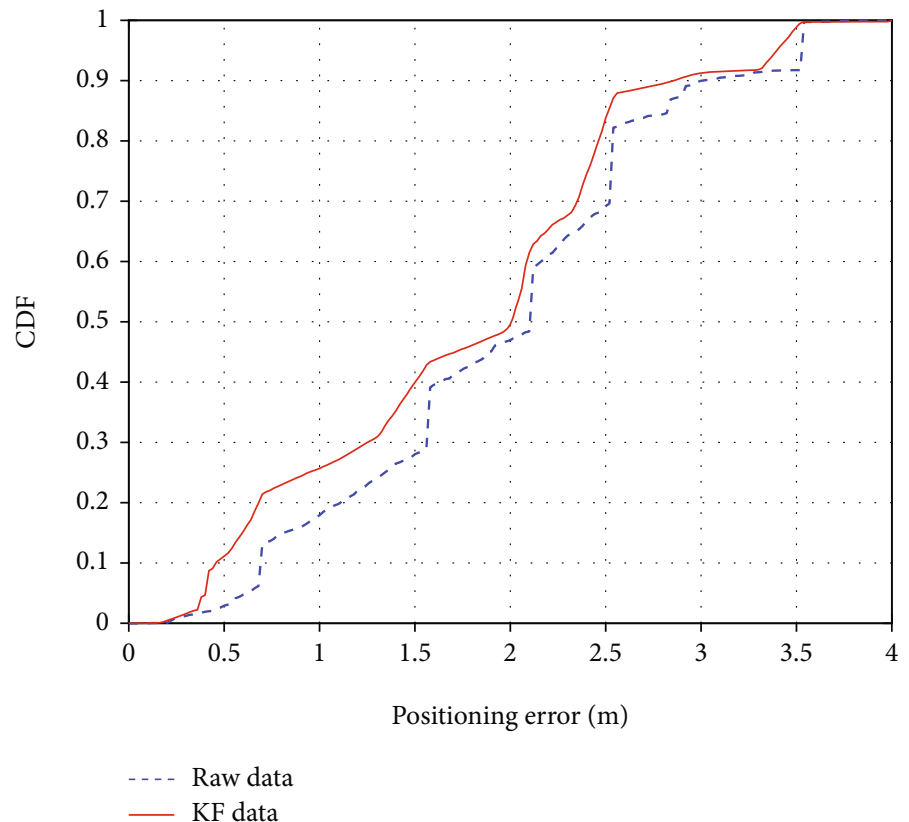

(b)

Figure 7: (a) Comparison of the Cumulative Distribution Function (CDF) of positioning error before and after Kalman filter in positioning room A. (b) Comparison of the CDF of positioning error before and after Kalman filter in positioning room B.

TABle 1: Positioning accuracy of room A.

\begin{tabular}{lccc}
\hline Location & Average error $(\mathrm{m})$ & CDF80\% $(\mathrm{m})$ & CDF90\% $(\mathrm{m})$ \\
\hline Raw data & 2.24 & 3.36 & 4.11 \\
KF data & 1.58 & 2.30 & 2.64 \\
\hline
\end{tabular}

TABle 2: Positioning accuracy of room B.

\begin{tabular}{lccc}
\hline Location & Average error $(\mathrm{m})$ & CDF80\% $(\mathrm{m})$ & CDF90\% $(\mathrm{m})$ \\
\hline Raw data & 1.95 & 2.54 & 3.08 \\
KF data & 1.77 & 2.41 & 2.85 \\
\hline
\end{tabular}



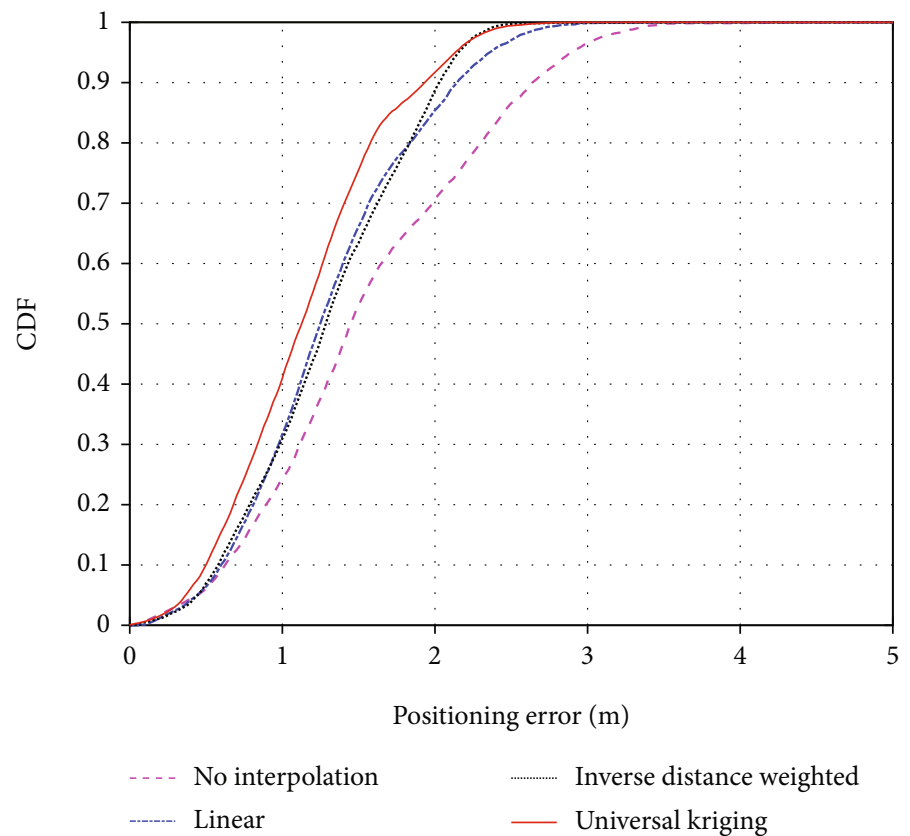

(a)

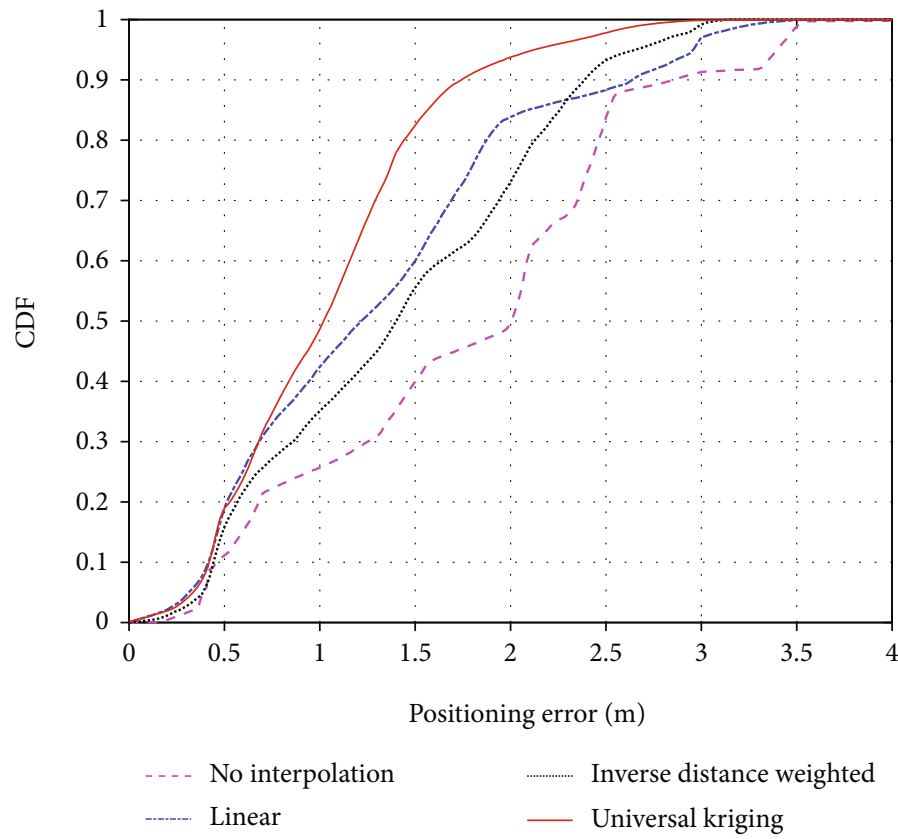

(b)

FIGURE 8: (a) CDF of the positioning error using different spatial interpolation algorithms in room A. (b) CDF of the positioning error using different spatial interpolation algorithms in room B.

$M_{k}$ and $M_{b}$ represent number of test points and samples over each point, respectively. All test samples equal to $M_{k} * M_{b}$.

5.2. RSS Preprocessing. To reduce the influence of varying multipath on RSS, Kalman filter is used to preprocess the NR RSS of the offline fingerprinting database and test points. The preprocessed RSS is more stable, and using KF effectively reduces the disturbance to NR RSS caused by varying multipaths. The RSS value changes slightly around the mean after preprocessing, the variance is smaller, and the data is more stable. Using KF to preprocess RSS is shown in Figure 6. The RSS comparison before and after preprocessing of reference point $\mathrm{A} 1$ in room $\mathrm{A}$ and $\mathrm{B} 1$ in room $\mathrm{B}$ is shown in Figures $6(\mathrm{a})$ and $6(\mathrm{~b})$, respectively. The distribution range of RSS values narrowed from $-58.4 \mathrm{dBm} \sim-50.3 \mathrm{dBm}$ to $-54.7 \mathrm{dBm} \sim-52.6 \mathrm{dBm}$, stabilizing around $-54 \mathrm{dBm}$. The distribution characteristics of RSS are more obvious.

In room A with a relatively simple room, the RSS distribution between each point is relatively close, and the characteristics are not obvious. After preprocessing, the RSS 
TABle 3: Positioning accuracy of room A after different interpolation algorithms.

\begin{tabular}{lccc}
\hline Location & Average error $(\mathrm{m})$ & $\begin{array}{c}\text { CDF80\% } \\
(\mathrm{m})\end{array}$ & $\begin{array}{c}\text { CDF90\% } \\
(\mathrm{m})\end{array}$ \\
\hline $\begin{array}{l}\text { No } \\
\text { interpolation }\end{array}$ & 1.58 & 2.30 & 2.64 \\
Linear & 1.32 & 1.84 & 2.14 \\
IDW & 1.30 & 1.84 & 2.03 \\
UK & 1.17 & 1.58 & 1.94 \\
\hline
\end{tabular}

TABle 4: Positioning accuracy of room B after different interpolation algorithms.

\begin{tabular}{lccc}
\hline Location & Average error $(\mathrm{m})$ & $\begin{array}{c}\text { CDF80\% } \\
(\mathrm{m})\end{array}$ & $\begin{array}{c}\text { CDF90\% } \\
(\mathrm{m})\end{array}$ \\
\hline $\begin{array}{l}\text { No } \\
\text { interpolation }\end{array}$ & 1.77 & 2.41 & 2.84 \\
Linear & 1.42 & 1.88 & 2.64 \\
IDW & 1.31 & 2.14 & 2.38 \\
UK & 1.16 & 1.44 & 1.76 \\
\hline
\end{tabular}

distribution of each point is more concentrated, so that the RSS cross-term between each point is reduced. The distribution characteristics of RSS between points are more obvious. We use the KNN positioning algorithm to locate the test points. As shown in Figure 7(a), the positioning accuracy is greatly affected by the multipath changes. Kalman filter on the raw RSS data significantly improves the positioning accuracy of the fingerprints. The positioning accuracy improvement effect is shown in Table 1. After using KF, the positioning accuracy has been improved by $31 \%$; we can achieve 2.30-meter positioning error for $80 \%$ test samples. The positioning error is the Euclidean distance of test points between the true position and the positioning position.

In room B, NR RSS value between each point varies greatly, and the distribution of the mean value is obvious. Varying multipath has little effect on the positioning accuracy. As shown in Figure 7(b), Kalman filter algorithm can improve the positioning accuracy. The positioning accuracy improvement effect is shown in Table 2. Using KF, the positioning accuracy has improved by $6 \%$. We achieve $2.41-$ meter positioning error for $80 \%$ test samples.

5.3. Spatial Interpolation. When we build the offline fingerprinting database in the fingerprinting positioning system, within a certain resolution range, the resolution of the offline fingerprinting database is proportional to the positioning accuracy. The higher the resolution of the fingerprints, the higher the positioning accuracy. Higher resolution will result in greater workload. In this experiment, a variety of commonly used spatial interpolation methods are used to assign values to each interpolation point separately. To avoid destroying the characteristics of the RSS of the interpolation points, we sort the RSS values of all points in descending order; the interpolation points are interpolated according to the weight of the reference points.
These experiments test different positioning environments in rooms A and B and compare several interpolation methods. We interpolate the preprocessed RSS offline fingerprinting database. The positioning error of various interpolation methods for rooms $A$ and $B$ is shown in Figure 8. In rooms $\mathrm{A}$ and $\mathrm{B}$, we use $\mathrm{UK}$ that has the best positioning accuracy. The interpolation accuracy in room $A$ is shown in Table 3, and the interpolation accuracy in room B is shown in Table 4. In room A, the positioning error of linear, IDW, and UK has improved by $20 \%, 20 \%$, and $31 \%$, respectively. Using UK can achieve 1.58-meter positioning error for $80 \%$ test samples. In room $\mathrm{B}$, the positioning error of linear, IDW, and UK has been improved by $24 \%, 11 \%$, and $40 \%$, respectively. Using UK can achieve 1.44-meter positioning error for $80 \%$ test samples. After using KF and UK, we can effectively improve the positioning accuracy in both rooms.

\section{Conclusions}

We use the existing $5 \mathrm{G}$ as the positioning base station, which need not rebuild specific positioning equipment. Our intelligent fingerprinting technology optimization adopts KF as preprocessing step to reduce the disturbance of the RSS values caused by multipath. Spatial interpolation method is used to keep fingerprint sampling effort low but still get good resolution. A variety of spatial interpolation methods are compared. UK has the best performance. In room A, compared with that of raw data, KF showed 31\% performance improvement. UK provided an additional $26 \%$ increase of positioning accuracy. In room $\mathrm{B}$, compared with that of raw data, KF has $6 \%$ performance improvement. UK can further reduce the positioning error by $36 \%$. We have achieved a positioning error of more than $80 \%$ test samples below 1.6 meters. In the next step, we may research universal fingerprinting, database preprocessing, and spatial interpolation methods in different indoor scenarios. Multiple-base station case in the future is possible. Research on maintaining the fingerprinting database over time is also good to touch.

\section{Data Availability}

The experimental data used to support the findings of this study are available from the corresponding author upon request.

\section{Conflicts of Interest}

The authors declare no conflict of interest.

\section{Acknowledgments}

This work was sponsored by the National Natural Science Foundation of China (No. 61771197) and Science and Technology Commission of Shanghai Municipality (Grant no. 18DZ2270800). 


\section{References}

[1] F. Zafari, A. Gkelias, and K. K. Leung, "A survey of indoor localization systems and technologies," IEEE Communications Surveys \& Tutorials, vol. 21, no. 3, pp. 2568-2599, 2019.

[2] S. A. Shaikh and A. M. Tonello, "Whitepaper on new localization methods for $5 \mathrm{G}$ wireless systems and the Internet-ofThings," in COST Action CA15104, European Cooperation in Science and Technology (COST), Brussels, Belgium, April 2018.

[3] K. Zhao, T. Zhao, Z. Zheng et al., "Optimization of time synchronization and algorithms with TDOA based indoor positioning technique for Internet of Things," Sensors, vol. 20, no. 22 , article $6513,2020$.

[4] X. Li, M. Zhao, M. Zeng et al., "Hardware impaired ambient backscatter NOMA systems: reliability and security," IEEE Transactions Communications, vol. 69, no. 4, pp. 2723-2736, 2021.

[5] C. Hua, K. Zhao, D. Dong et al., "Multipath map method for TDOA based indoor reverse positioning system with improved Chan-Taylor algorithm," Sensors, vol. 20, no. 11, article 3223, 2020.

[6] B. Wang, X. Liu, B. Yu, R. Jia, and X. Gan, “An improved WiFi positioning method based on fingerprint clustering and signal weighted Euclidean distance," Sensors, vol. 19, no. 10, article 2300, 2019.

[7] C.-Y. Chen and W.-R. Wu, "Three-dimensional positioning for LTE systems," IEEE Transactions on Vehicular Technology, vol. 66, no. 4, pp. 3220-3234, 2017.

[8] C.-H. Wang, C.-J. Lee, and X. Wu, "A coverage-based location approach and performance evaluation for the deployment of 5G base stations," IEEE Access, vol. 8, pp. 123320-123333, 2020.

[9] B. el Boudani, L. Kanaris, A. Kokkinis et al., "Implementing deep learning techniques in $5 \mathrm{G}$ IoT networks for $3 \mathrm{D}$ indoor positioning: DELTA (DeEp Learning-Based Co-operaTive Architecture)," Sensors, vol. 20, no. 19, article 5495, 2020.

[10] V. Savic and E. G. Larsson, "Fingerprinting-based positioning in distributed massive MIMO systems," in 2015 IEEE 82nd Vehicular Technology Conference (VTC2015-Fall), pp. 1-5, Boston, MA, USA, September 2015.

[11] X. Li, J. Li, Y. Liu, Z. Ding, and A. Nallanathan, "Residual transceiver hardware impairments on cooperative NOMA networks," IEEE Transactions on Wireless Communications, vol. 19, no. 1, pp. 680-695, 2020.

[12] E. Rastorgueva-Foi, M. Costa, M. Koivisto, K. Leppänen, and M. Valkama, "User positioning in mmW 5G networks using beam-RSRP measurements and Kalman filtering," in 2018 21st International Conference on Information Fusion (FUSION), pp. 1-7, Cambridge, UK, July 2018.

[13] X. Li, M. Zhao, Y. Liu, L. Li, Z. Ding, and A. Nallanathan, "Secrecy analysis of ambient backscatter NOMA systems under I/Q imbalance," IEEE Transactions on Vehicular Technology, vol. 69, no. 10, pp. 12286-12290, 2020.

[14] M. A. Khan, N. Saeed, A. W. Ahmad, and C. Lee, "Location awareness in $5 \mathrm{G}$ networks using RSS measurements for public safety applications," IEEE Access, vol. 5, pp. 21753-21762, 2017.

[15] F. Wang, J. Chen, and Q. Liu, "SRS-based LTE indoor wireless positioning system," in 2017 IEEE 2nd Advanced Information Technology, Electronic and Automation Control Conference (IAEAC), pp. 2356-2359, Chongqing, China, March 2017.
[16] C. Zhou, J. Yuan, H. Liu, and J. Qiu, "Bluetooth indoor positioning based on RSSI and Kalman filter," Wireless Personal Communication, vol. 96, no. 3, pp. 4115-4130, 2017.

[17] S. Kram, C. Nickel, J. Seitz, L. Patino-Studencka, and J. Thielecke, "Spatial interpolation of Wi-Fi RSS fingerprints using model-based universal kriging," in 2017 Sensor Data Fusion: Trends, Solutions, Applications (SDF), pp. 1-6, Bonn, Germany, October 2017.

[18] S. Sam and C. James, "Reducing the effect of signal multipath fading in RSSI-distance estimation using Kalman filters," in 19th Communications \& Networking Symposium (CNS 2016), Pasadena, CA, USA, 2016.

[19] Y. Zhuang, Y. Li, L. Qi, H. Lan, J. Yang, and N. el-Sheimy, "A two-filter integration of MEMS sensors and WiFi fingerprinting for indoor positioning," IEEE Sensors Journal, vol. 16, no. 13, pp. 5125-5126, 2016.

[20] J. Zuo, S. Liu, H. Xia, and Y. Qiao, "Multi-phase fingerprint map based on interpolation for indoor localization using iBeacons," IEEE Sensors Journal, vol. 18, no. 8, pp. 3351-3359, 2018.

[21] A. H. Ismail, H. Kitagawa, R. Tasaki, and K. Terashima, "WiFi RSS fingerprint database construction for mobile robot indoor positioning system," in 2016 IEEE International Conference on Systems, Man, and Cybernetics (SMC), pp. 1561-1566, Budapest, Hungary, October 2016.

[22] P.-W. Son, J. H. Rhee, J. Hwang, and J. Seo, "Universal kriging for Loran ASF map generation," IEEE Transactions on Aerospace and Electronic Systems, vol. 55, no. 4, pp. 1828-1842, 2019.

[23] T. Koike-Akino, P. Wang, M. Pajovic, H. Sun, and P. V. Orlik, "Fingerprinting-based indoor localization with commercial MMWave WiFi: a Deep learning approach," IEEE Access, vol. 8, pp. 84879-84892, 2020.

[24] A. Belmonte-Hernandez, G. Hernandez-Penaloza, D. Martin Gutierrez, and F. Alvarez, "SWiBluX: multi-sensor deep learning fingerprint for precise real-time indoor tracking," IEEE Sensors Journal, vol. 19, no. 9, pp. 3473-3486, 2019.

[25] C. Du, B. Peng, Z. Zhang, W. Xue, and M. Guan, "KF-KNN: low-cost and high-accurate FM-based indoor localization model via fingerprint technology," IEEE Access, vol. 8, pp. 197523-197531, 2020.

[26] H. Zhao, B. Huang, and B. Jia, “Applying kriging interpolation for WiFi fingerprinting based indoor positioning systems," in 2016 IEEE Wireless Communications and Networking Conference, pp. 1-6, Doha, Qatar, April 2016. 